\title{
Approximate Coverage in Wireless Sensor Networks
}

\author{
Yuzhen Liu Weifa Liang \\ Department of Computer Science \\ The Australian National University \\ Canberra, ACT 0200, Australia \\ Email: \{yliu,wliang\}@cs.anu.edu.au
}

\begin{abstract}
Recent advances in microelectronic technology have made it possible to construct compact and inexpensive wireless sensors. Sensor networks have received significant attention due to their potential applications from civil to military domains. Since sensors in sensor networks are equipped with energylimited batteries, energy conservation in such networks is of paramount importance in order to prolong the network lifetime.

Sensing coverage and sensor connectivity in sensor networks are two fundamental issues, which have been extensively addressed in the literature, and most existing work on sensing coverage has focused on the (connected) full coverage problem that aims to cover the entire monitored region using the minimum number of sensors. However, in some application scenarios, full coverage is either impossible or unnecessary and a partial coverage with a certain degree guarantee is acceptable. In this paper, we study the connected coverage problem with a given coverage guarantee. We first introduce the partial coverage concept and analyze its properties for the first time in order to prolong the network lifetime. Due to NP-hardness of the concerned problem, we then present a heuristic algorithm which takes into account the partial coverage and sensor connectivity simultaneously. We finally conduct extensive experiments by simulations to evaluate the performance of the proposed algorithm.
\end{abstract}

\section{INTRODUCTION}

Recent advances in microelectronic technology have made it possible to construct compact and inexpensive wireless sensors. Networks formed by such sensors, termed as wireless sensor networks, combine simple wireless communication, minimal computation facilities, and some sort of sensing of the physical environment into the new form of network, which have received significant attention due to their potential applications from civil to military domains including environment monitoring, biological detection, vehicle tracking, and battlefield surveillance [1]. Sensor networks are used to collect sensed data, aggregate them if needed, and transmit them to the base station. The sensors in such networks are deployed in a region of interest and their locations are unknown a priori. Each sensor serves not only as a host to sense data around its vicinity but also as a router to relay messages for other sensors toward the base station. One critical characteristic of a sensor network is that each sensor in it is equipped with energylimited battery, and energy-efficiency in network operations is of paramount importance.

One important problem that arises in high-density sensor networks is density control - a function that controls the density of working sensors to a certain level [15]. Specifically, density control ensures only a subset of sensors operates in active mode. Also, not only should the sensors in the chosen set be able to cover the monitored region with a given coverage guarantee but the communication graph induced by the sensors should be connected. Thus, sensing coverage and sensor connectivity in sensor networks are two fundamental issues. Sensor networks are typically used to monitor a region of interest. The sensors can cover the partial or entire region, depending on application domains. The ratio between the covered area by sensors and the entire monitored region is an important metric in terms of the quality of service of sensing coverage, i.e. how accurate the sensor network monitors the region of interest, how far away a point in an uncovered area is from its nearest active sensor. The full coverage problem, in which each point in the entire monitored region is covered by at least one sensor, has been extensively studied in literature [2], [3] [5]-[17]. However, in some applications, full coverage may not be necessary and partial coverage is sufficient. Since the number of sensors used for partial coverage is significantly less than that for full coverage, the network lifetime by partial coverage is further prolonged through keeping a small number of sensors in active mode. One such an example is the environment monitoring. It usually requires an approximate (partial) coverage for the monitored region. Only when an abnormality occurs, we may increase the coverage accuracy to fully coverage by checking the details of the abnormality (called zoom-in and zoom-out approach). We refer to this latter problem as the partial coverage problem, which is defined as follows. Given a monitored region with densely deployed sensors, the problem is to find a subset of sensors and keep them in active mode while the other sensors are kept in sleep mode so as to preserve their precious energy, in order to prolong the network lifetime and to meet the partial (full) coverage guarantee simultaneously. On the other hand, the sensed data by active sensors must be transmitted to the base station for further processing. To do so, it requires that the selected sensors be able to communicate with each other, directly or through relay nodes. Therefore, our objective is to not only find a subset of sensors for partial coverage with a given coverage guarantee but also ensure that the communication graph induced by the chosen sensors is connected, we refer to this as the connected, partial coverage problem. One of its special cases - the connected, full coverage problem has been explored in [5], [7], [8], [9], [12], [16], [17].

\section{A. Related Work}

In recent years, tremendous effort in wireless sensor networks has been taken on covering the monitored racinn preserving network connectivity, maximizing energy eft

(LCN'05) 
and network lifetime [2]-[17]. Among them, [4] surveyed the recent progress of the full coverage and connectivity problem.

Given a wireless sensor network, Meguerdichian et al [8] considered the coverage as the measure of quality of service of a sensor network. They presented algorithms for solving the worst (breach) and best (support) coverage by proposing the maximal breach path which has the property that the distance from a point $p$ to its nearest sensor is maximized, and the maximal support path which has the property that the distance from a point $p$ to its nearest sensor is minimized, where $p$ is a point on the path. For the full coverage problem, Slijepcevic and Potkonjak [10] initially studied this problem by proposing an approach that partitions the sensors in the network into mutually exclusive subsets such that the sensors in each subset can fully cover the monitored region, and their goal is to maximize the number of the subsets. Thus, it suffices to have the sensors in one subset to be active at any time. A heuristic is then proposed for sensor partitioning due to the NP-hardness of the concerned problem. However, they focused only on full coverage without taking into account the connectivity of sensors in each subset. Furthermore, the input of their algorithm is the set of fields, while finding such a field partition is time-consuming and difficult in practice, where a field is a set of points that are covered by the same set of sensors. Cardei et al [2] considered full coverage by proposing an approximation algorithm for partitioning the sensors into the maximum number of disjoint dominating sets, where each disjoint dominating set can fully cover the monitored region. Thus, only one of the disjoint dominating sets is active and responsible for the monitored region at any given time. Their objective aims to achieve better energy savings, but there may exist a point in the monitored region that is not covered by any sensor in the dominating set. Tian and Georganas [11] considered the fully coverage problem as well by exploring an energy-efficient coverage-preserving node-scheduling scheme to prolong the network lifetime.

Gupta et al [7] studied the network connectivity and full coverage (the connected, full coverage problem) for spatial queries by introducing the concept of connected sensor cover. They proposed centralized and distributed self-organization algorithms to select a subset of sensors that suffices to a spatial query processing in a given region. Zhou et al [17] generalized the connected, full coverage problem into the connected $k$ that each point in the monitored region is covered by at least $k$ sensors in the set of selected sensors. Zhang and Hou [16] addressed the problem of maintaining sensing coverage and connectivity, with an objective to minimize the number of active sensors. With an assumption that the transmission range $r_{c}$ of each sensor is at least twice its sensing range $r_{s}$, they proved that if a set of sensors fully covers the entire region, then the communication graph induced by the sensors is connected. Thus, they only consider the coverage problem under the assumption by proposing a distributed algorithm for the connected, full coverage problem. They also considered the case of $r_{c}<2 r_{s}$ by giving a heuristic algorithm as well. Wang et al [12] extended the approach in [16] to solve the $k$-coverage problem by showing that $k$-coverage implies $k$ coverage problem, which aims at finding a set of sensors such

connectivity under the same assumption, where a graph is $k$-connected if it is still connected after the removal of any $k-1$ nodes from it. It must be mentioned that although Zhang and Hou [16] showed with an assumption of $r_{c} \geq$ $2 r_{s}$ that if a set of sensors fully covers a given monitored region, then the communication graph induced by the sensors is connected, this property, however, is no longer held if it is partial rather than full coverage. It must be mentioned that a similar notion to partial coverage in this paper was proposed in [16], which is described as follows. Initially it is expected to obtain full coverage. After a certain number of rounds, it is impossible to obtain a full coverage using active sensors, due to the expiration of energy of some sensors, instead, a partial coverage with a given threshold is acceptable. The connected, partial coverage problem considered in this paper is essentially different from theirs in the following aspects. (i) Given a coverage guarantee $\theta$, we aim at covering $\theta$ percentage of the monitored region using the minimum number of sensors, whereas in [16], they tried to cover the entire region fully at first, and then reduce the coverage percentage to a given threshold provided it is impossible to have a full coverage by the living sensors. Thus, more sensors than actually needed are selected as active sensors in the beginning of the network operation. (ii) We aim to partially cover the monitored region evenly and guarantee that the distance between a point that is not covered by any selected sensor and its nearest active sensor is within a given threshold, depending on the application requirement, whereas in [16], they consider neither the distribution of covered areas nor the bound on the above distance, and thus partial coverage obtained by their approach may be unfair, i.e. some areas are covered while other reasonably large areas may not be covered. (iii) In our approach, the communication graph induced by the selected sensors is guaranteed to be connected, whereas the graph induced by the selected sensors in [16] may be disconnected.

\section{B. Motivations}

Most existing work on sensing coverage in the literature has focused on the (connected) full coverage problem. However, in some scenarios, full coverage is either impossible or unnecessary. One such a scenario is the sensor network for weather forecasting, in which the sensed data is satisfactory if the coverage percentage of the monitored region is no less than a given threshold, e.g. $90 \%$. On the other hand, because of random deployment of sensors, it cannot be guaranteed that the deployed sensors are able to cover the entire region. In addition, some sensors in a sensor network may become invalid due to the expiration of their energy or the failure to communicate with the other sensors. Instead, it is usually acceptable that the deployed sensors cover only partial monitored region with a given coverage guarantee. That is, partial coverage is more realistic than full coverage in some harsh environments. Specifically, for a given coverage guarantee $\theta$, the partial coverage problem is to select a set of sensors with minimum cardinality such that the chosen sensors can merer at least $\theta$ percentage of the entire region. The valu (LCN'05)

Proceedings of the IEEE Conference on Loc
$0-7695-2421-4 / 05 \$ 20.00$ (C) 2005 IEEE
COMPUTER SOCIETY 
represents the accuracy of the sensed data in some degree, depending on application domains, where $0<\theta \leq 1$. Due to the nature of partial coverage, it is inevitable that there are some areas in the monitored region in which no point can be covered by any chosen sensor. We refer to each of these uncovered areas as a sensing void (or $S V$ for short). Therefore, the distribution of the covered areas and the distance between a point in a sensing void and its nearest active sensor are important metrics in terms of the quality of partial coverage, which will determine the coverage accuracy.

\section{Contributions}

In this paper, we first propose the concept of partial coverage in sensor networks for the first time (referred to as $\theta$-coverage, $0<\theta \leq 1$ ) for further reducing energy consumption, thereby prolonging the network lifetime. We then analyze the properties of partial coverage and present a heuristic algorithm that takes partial coverage and connectivity into consideration simultaneously. We finally conduct extensive experiments by simulation to evaluate the performance of the proposed algorithm.

The rest of the paper is organized as follows. In Section II the wireless sensor network model and problem definitions are introduced. In Section III the properties of partial coverage are analyzed. In Section IV a heuristic algorithm for the connected, partial coverage problem is proposed. In Section V, to evaluate the performance of the proposed algorithm, extensive experiments by simulation are conducted. In Section VI the conclusion is given.

\section{PRELIMINARIES}

\section{A. System Model}

We consider a wireless sensor network consisting of $n$ sensor nodes randomly deployed on a region of interest. Each sensor has an omnidirectional antenna and can monitor its vicinity. In other words, a wireless sensor network can be modelled by an undirected communication graph $G=(N, E)$, where $N$ is the set of sensor nodes with $|N|=n$, and there is an undirected edge $(u, v)$ in $E$ if and only if nodes $u$ and $v$ are within the transmission ranges of each other with $|E|=m$. A simple path $P_{u, v}$ in $G$ between $u$ and $v$ is a sequence of nodes $v_{0}, v_{1}, v_{2}, \cdots, v_{t}$, where $u=v_{0}, v=v_{t}, v_{i} \in N$, $\left(v_{j}, v_{j+1}\right) \in E, 0 \leq i \leq t$ and $0 \leq j<t$. The length of $P_{u, v}$, referred to as $\left|P_{u, v}\right|$, is the number of nodes in it. $G$ is connected if there is a simple path in $G$ between $u$ and $v$ for each pair of nodes $u$ and $v, u, v \in N$. Assume $N^{\prime} \subseteq N, G^{\prime}=\left(N^{\prime}, E^{\prime}\right)$ is an induced subgraph of $G$ by $N^{\prime}$ if $E^{\prime}=E \cap\left(N^{\prime} \times N^{\prime}\right)$.

In order to cover a monitored region $R$ "evenly", we assume that $R$ is partitioned into a number of equal-size blocks, and use the terminology area to represent a region or a block. The coverage area function $\mathcal{C}$ of an area $A$, referred to as $\mathcal{C}(A)$, is the area of $A$. The intersection of two areas $A_{1}$ and $A_{2}$, referred to as $A_{1} \cap A_{2}$, is the overlapping part of $A_{1}$ and $A_{2}$.

A sensor is used to monitor its vicinity. The sensing field of sensor $s$, referred to as $f_{s}(s)$, is the well-defined (convex) area around itself. The field $f_{s}(s)$ is covered by sensor $s$, and any point in $f_{s}(s)$ is covered by $s$. A point is covered by a set $S$ of sensors if at least one sensor in $S$ covers it. If $f_{s}(s)$ is a disk, the sensing range of $s$, referred to as $r_{s}(s)$, is the radius of the disk. The transmission range of sensor $s$, referred to as $r_{c}(s)$, is the maximum distance less than or equal to which it can communicate with the other sensors. In this paper, we use $f_{s}, r_{s}$ and $r_{c}$ to denote $f_{s}(s), r_{s}(s)$ and $r_{c}(s)$ respectively for simplicity, due to the fact that we assume the sensors in a sensor network are homogeneous. Note that the proposed algorithm can also be applied to the case where sensors in a sensor network are heterogeneous.

\section{B. Partial Sensor Coverage}

We now introduce the concept of partial sensor cover, which is different from the full sensor cover in the literature in which the selected sensors cover the entire monitored region. For a given coverage guarantee $\theta$ with $0<\theta \leq 1$, the partial sensor cover is a set of sensors with minimum cardinality that covers at least $\theta$ percentage of the entire region. The partial sensor cover is also referred to as the $\theta$-cover. A special case of partial cover is the full sensor cover when $\theta=1$. To distribute the covered areas throughout the entire monitored region evenly, the monitored region can be partitioned into a number of equal-size blocks. This leads to the evenly partial sensor cover, which is a set of sensors with minimum cardinality such that each of the blocks in the monitored region is $\theta$ covered. In some applications, it may still be insufficient to have the monitored region been either fully or partially covered, because the communication graph induced by the sensors in the partial or full cover might not be connected, while the sensed data by the sensors in the network must be transmitted to the base station through the sensors. Thus, it is required that the sensors in a partial or full sensor cover be able to communicate with each other so that the sensed data can be transmitted to the base station. If the communication graph induced by a full (partial or evenly partial) sensor cover is connected, the sensor cover is referred to as a connected, full (partial or evenly partial) sensor cover. In the following, we formally define the concept of partial sensor cover.

Let $S$ be a set of sensors deployed in a given monitored region $R$ that has been partitioned into equal-size blocks $Q_{1}, Q_{2}, \cdots, Q_{t}, t \geq 1$, and $\theta$ the coverage guarantee with $0<\theta \leq 1$. $Q_{i}$ is $\theta$-covered by $S$, or $S$ is a $\theta$-cover of $Q_{i}$, if there is a subset $S^{\prime} \subseteq S$ such that the area of the intersection of $Q_{i}$ and the union of the sensing fields of sensors in $S^{\prime}$ is not less than $\theta$ percentage of the area of $Q_{i}$, i.e. $\exists_{S^{\prime} \subseteq S}\left(\mathcal{C}\left(Q_{i} \cap\left(\cup_{s \in S^{\prime}} f_{s}\right)\right) \geq \theta \mathcal{C}\left(Q_{i}\right)\right), 1 \leq i \leq t$. $R$ is $\theta-$ covered by $S$, or $S$ is a $\theta$-cover of $R$ if there exists a subset $S^{\prime} \subseteq S$ such that the area of the intersection of $R$ and the union of the sensing fields of sensors in $S^{\prime}$ is not less than $\theta$ percentage of the area of $R$, i.e. $\exists_{S^{\prime} \subseteq S}\left(\mathcal{C}\left(R \cap\left(\cup_{s \in S^{\prime}} f_{s}\right)\right) \geq\right.$ $\theta \mathcal{C}(R)) . R$ is evenly $\theta$-covered by $S$, or $S$ is a evenly $\theta$-cover of $R$ if each of its blocks is $\theta$-covered by $S$. If the subgraph induced by $S^{\prime}$ is connected, $S$ is a connected $\theta$-cover of $Q_{i}$, a connected $\theta$-cover of $R$, or a connected, evenly $\theta$-cmor of $R$ respectively. 


\section{Problem Definitions}

Let $S$ be the set of sensors deployed in a given monitored region $R$ that has been partitioned into equal-size blocks $Q_{1}, Q_{2}, \cdots, Q_{t}, t \geq 1$, and $\theta$ the coverage guarantee with $0<\theta \leq 1$. The $\theta$-coverage problem is to find a subset $A S$ of $S$ with minimum cardinality such that $R$ is $\theta$-covered by $A S$. Note that although the sensors in $A S$ for the $\theta$ coverage problem can cover $\theta$ percentage of $R$, the sensors in $A S$ may not be able to communicate with each other. The connected $\theta$-coverage problem is to find a subset $A S$ of $S$ with minimum cardinality such that $R$ is $\theta$-covered by $A S$ and the communication graph induced by $A S$ is connected. Furthermore, even if $R$ is connected $\theta$-covered by a set $A S$ of sensors, it is possible that there may exist a large sensing void in $R$. As shown in Fig.1(a) the top white space is unexpected and will reduce the sensing accuracy. Thus, it is desirable that the covered areas by the sensors in $A S$ be evenly distributed throughout $R$. In other words, the un-sensed areas should be distributed in $R$ evenly. The connected, evenly $\theta$-coverage problem thus is to find a subset $A S$ of $S$ with minimum cardinality such that each block of $R$ is $\theta$-covered by $A S$ and the communication graph induced by $A S$ is connected. The sensors in $A S$ are known as active sensors.

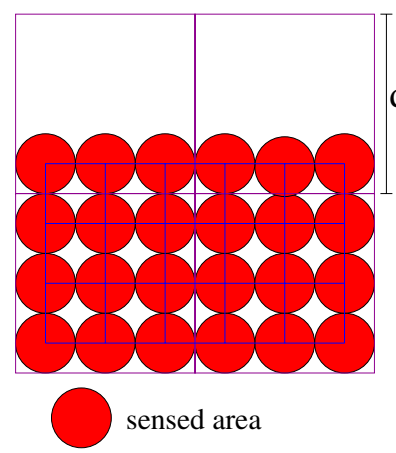

(a)

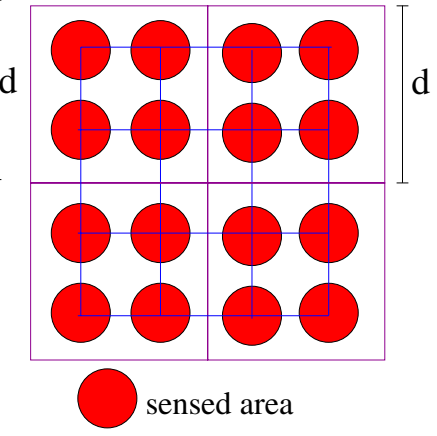

(b)
Fig. 1. (a)The large sensing void; (b)The maximum sensing void.

In this paper, we will deal with the connected, evenly $\theta$ coverage problem. A special case of the concerned problem when $\theta=1$ has been well addressed in [5], [7], [9], [12], [16]. Also, it is well known that the connected, full coverage problem is NP-Complete, so is the connected, evenly $\theta$ coverage problem.

To evaluate the quality of a given solution to this problem, a typical measurement is to calculate the area of each sensing void. Intuitively, the smaller a sensing void is, the better the solution is. Clearly, this measurement aims to avoid large sensing voids in $R$. However, we argue that this measurement is insensible because it is inevitable that there is large sensing void due to the nature of partial coverage. This can be seen through an example illustrated in Fig.1(b), where the area of a sensing void induced by an evenly partial sensor cover can be as large as $(1-\theta) \mathcal{C}(R)$. Furthermore, this measurement does not take into consideration the irregularities of shapes of sens- ing voids. Instead, we propose here a sensible measurement for sensing voids. That is, we measure the distance between a point in a sensing void and its nearest active sensor. The shorter the distance is, the better the solution is, because the shorter distance reflects that the point is not far away from its nearest active sensor.

Let $S V$ be a sensing void and $p$ a point in $S V$. The gap of $p$ in $S V$ relative to $A S$, referred to as $\operatorname{gap}(p, A S)$, is the distance between $p$ and its nearest sensor in $A S$, i.e. $\operatorname{gap}(p, A S)=$ $\min \{\operatorname{distance}(p, s) \mid s \in A S\}$, where $\operatorname{distance}(p, s)$ is the Euclidean distance between $p$ and $s$. The gap of $S V$ relative to $A S$, referred to as $\operatorname{gap}_{S}(S V, A S)$, is the maximum among all $\operatorname{gap}(p, A S)$ for any point $p$ in $S V$, i.e. $\operatorname{gap}_{S}(S V, A S)=$ $\max \{\operatorname{gap}(p, A S) \mid p \in S V\}$.

\section{Properties of Partial Coverage}

In this section we first explore several important properties of partial coverage and then analyze the quality of the partial coverage.

Observation For a given monitored region $R$, a set $S$ of sensors, a coverage guarantee $\theta$, and the sensing range $r_{s}$ of each sensor, the minimum number of sensors for the $\theta$ coverage problem is $N_{\text {opt }}(\theta) \geq\left\lceil\frac{\theta \mathcal{C}(R)}{\pi r_{s}^{2}}\right\rceil$, where $0<\theta \leq 1$.

It is obvious because each sensor covers at most $\pi r_{s}^{2}$ area. Notice that this observation only provides a lower bound on the number of sensors required for the $\theta$-coverage problem. It, however, does not ensure that the communication graph induced by these sensors is connected.

Lemma 1: For a coverage guarantee $\theta$ with $0<\theta \leq 1$, a set $S$ of sensors with sensing range $r_{s}$ and communication range $r_{c}$, assume that a given a monitored region $R$ has been connected $\theta$-covered, then, no sensing void can partition the covered area in $R$ if $r_{c} \leq 2 r_{s}$. In other words, there is only one covered area.

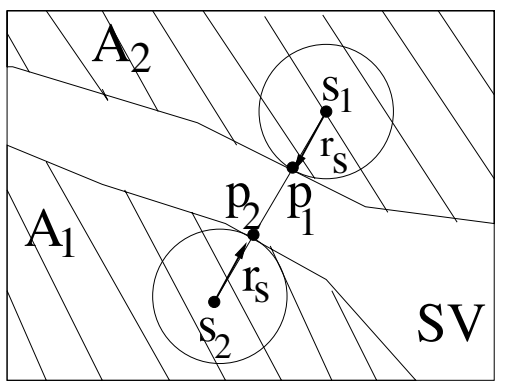

Fig. 2. Two covered areas partitioned by a sensing void

Proof: We show the claim by contradiction. We assume that the solution of the connected $\theta$-coverage problem is $A S$, $A_{1}$ and $A_{2}$ are two covered areas which are separated by a sensing void $S V$ as shown in Fig.2. We further assume that $A_{1}$ and $A_{2}$ are covered by sensors in $A S_{1}$ and $A S_{2}$, where $A S_{1} \cup A S_{2} \subseteq A S$ and $A S_{1} \cap A S_{2}=\emptyset$. Let $s_{1}$ and $s_{2}$ be such two sensors in $A S_{1}$ and $A S_{2}$ respectively that the distance between them is the minimum one among any pair of sensors $s_{i} \in A S_{1}$ and $s_{j} \in A S_{2}$, i.e. distance $\left(s_{1} c_{n}\right)-$ $\min \left\{\operatorname{distance}\left(s_{i}, s_{j}\right) \mid s_{i} \in A S_{1}, s_{j} \in A S_{2}\right\}$. A strai (LCN'05) 
connecting them is drawn. Let $p_{i}$ be the intersecting point of the straight line and the boundary of the sensing field $f_{s_{i}}$ of $s_{i}, i=1,2$, illustrated in Fig.2. Then, distance $\left(s_{1}, s_{2}\right)=$ $2 r_{s}+\overline{p_{1} p_{2}}>2 r_{s} \geq r_{c}$, due to $\overline{p_{1} p_{2}}>0$. Thus, none of sensors in $A S_{1}$ can communicate with any sensor in $A S_{2}$, because $\operatorname{distance}\left(s_{i}, s_{j}\right) \geq \operatorname{distance}\left(s_{1}, s_{2}\right)>r_{c}$, for any pair of sensors $s_{i} \in A S_{1}$ and $s_{j} \in A S_{2}$. Therefore, the induced graph by $A S$ is disconnected, which contradicts the fact that $R$ is connected $\theta$-covered.

A monitored region $R$ is densely deployed by sensors if a sensor can be found at any desirable point. We assume that $R$ is large enough so that the boundary effect of $R$ can be ignored. In the following we show a tight bound on the minimum number of sensors for the connected, evenly $\theta$ coverage problem. The idea behind this is that we first partition $R$ into a number of equal-size hexagons such that none of any two hexagons are overlapped. We then draw an interior circle at each hexagon which is the sensing field by a sensor located at the center. The edge length $h$ of each hexagon is chosen such that $\frac{\text { the area of the interior circle }}{\text { the area the hexagon }} \geq \theta$. We state this in the following theorem.

Theorem 1: For a given densely deployed monitored region $R$ which is large enough so that the boundary effect of $R$ can be ignored, the coverage guarantee $\theta$, the sensing range $r_{s}$ and the transmission range $r_{c}$ of each sensor, the tight bound on the number of active sensors for the connected, evenly $\theta$-coverage problem is $\left\lceil\frac{\theta \mathcal{C}(R)}{\pi r_{s}^{2}}\right\rceil$ if $r_{c} \geq 3^{-\frac{1}{4}} \sqrt{\frac{2 \pi}{\theta}} r_{s}$, where $0<\theta \leq \frac{\sqrt{3} \pi}{6}$.

Proof: The monitored region $R$ is first partitioned into a

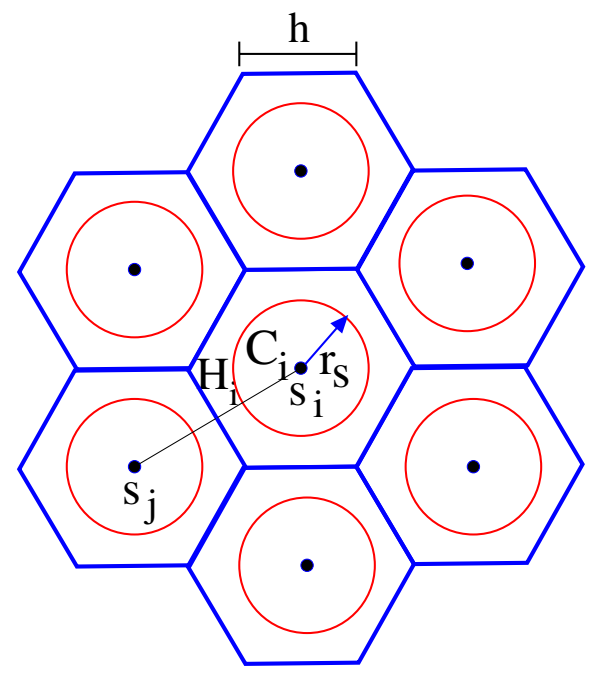

Fig. 3. The tight bound of the number of sensors

number of equal-size hexagons $H_{1}, H_{2}, \cdots, H_{l}(l \geq 1)$ such that the edge length of a hexagon is $h=3^{-\frac{3}{4}} \sqrt{\frac{2 \pi}{\theta}} r_{s}$. For each $H_{i}$, there is a sensor $s_{i}$ at its barycenter since $R$ is densely deployed. An interior circle $C_{i}$ centered at $s_{i}$ with radius $r_{s}$ is then drawn, as shown in Fig.3. Then, $H_{i}$ is $\theta$-covered by its interior circle $C_{i}$ because $\frac{\mathcal{C}\left(C_{i}\right)}{\mathcal{C}\left(H_{i}\right)}=\frac{\pi r_{s}^{2}}{\frac{3 \sqrt{3}}{2} h^{2}}=\theta, 1 \leq i \leq l$. As a result, $R$ is evenly $\theta$-covered by $\left\{s_{i} \mid 1 \leq i \leq l\right\}$, and the value of $\theta$ is maximized when $C_{i}$ becomes the inscribed circle, where $r_{s}=\frac{\sqrt{3} h}{2}, \theta=\frac{\pi r_{s}^{2}}{3 h r_{s}}=\frac{\sqrt{3} \pi}{6}$. Since there is no overlapping between the sensing fields of any two sensors, the tight bound on the number of sensors for the evenly $\theta$-coverage problem is $\left\lceil\frac{\theta \mathcal{C}(R)}{\pi r_{s}^{2}}\right\rceil$.

The rest is to show that the communication graph induced by $\left\{s_{i} \mid 1 \leq i \leq l\right\}$ is connected. The distance between the incenters of interior circles within any two neighboring hexagons is $\sqrt{3} h=3^{-\frac{1}{4}} \sqrt{\frac{2 \pi}{\theta}} r_{s}$. Thus, the sensors located at the incenters of interior circles of hexagons form a connected communication graph if $r_{c} \geq 3^{-\frac{1}{4}} \sqrt{\frac{2 \pi}{\theta}} r_{s}$.

The $\theta$-coverage problem is a special case of the evenly $\theta$ coverage problem when $R$ consists of one block only. We focus on the connected, evenly $\theta$-coverage problem. We bound the gap of sensing voids by the following theorem.

Theorem 2: For a given coverage guarantee $\theta$ with $0<$ $\theta<1$, a monitored region $R$ that has been partitioned into a number of equal-size blocks with edge length of $d$ and is evenly $\theta$-covered by $S$ with the set $A S$ of active sensors, the sensing range $r_{s}$ of each sensor, the sensor voids $S V_{i}(1 \leq i \leq q)$, and a point $p \in \cup_{1 \leq i \leq q} S V_{i}$, we have (1) $r_{s}<\operatorname{gap}(p, A S) \leq 2 d \sqrt{\frac{1-\theta}{\pi}}+r_{s} ;(2) r_{s}<\operatorname{gap}_{S}\left(S V_{i}, A S\right) \leq$ $2 d \sqrt{\frac{1-\theta}{\pi}}+r_{s}$, for all $i, 1 \leq i \leq q$.

Proof: Following the definition of $\operatorname{gap}(p, A S)$, it is easy to see that $r_{s}<\operatorname{gap}(p, A S)$. Otherwise, $p$ will be covered by a sensor in $A S$. This contradicts the fact that $p$ is a point in a sensing void $S V_{i}, 1 \leq i \leq q$.

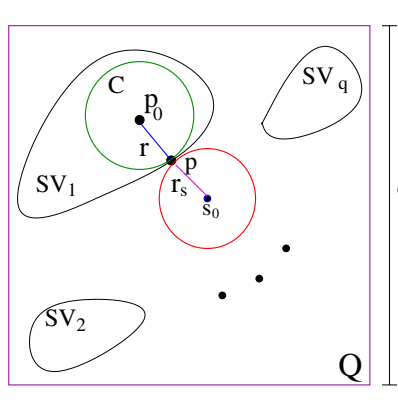

(a)

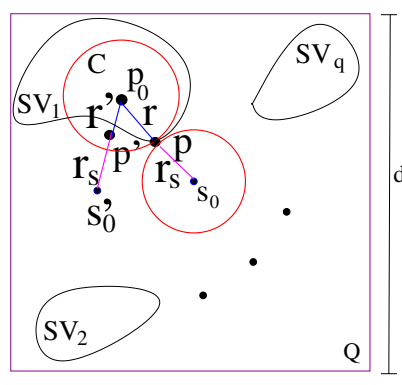

(c)

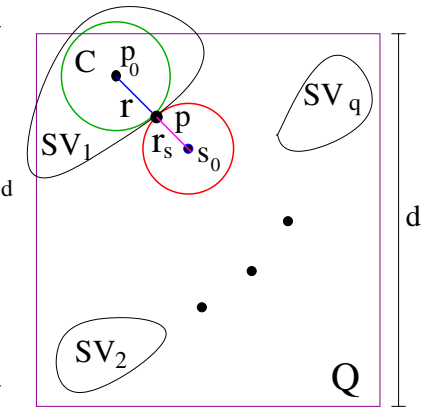

(b)

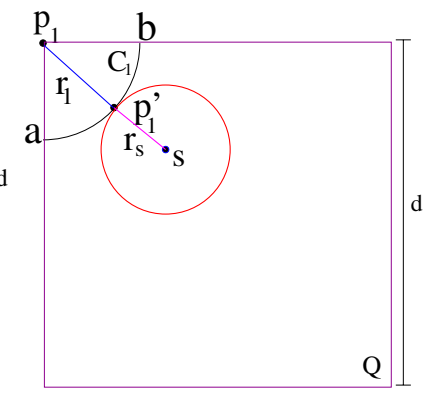

(d)
Fig. 4. Four cases on the upper bound of $\operatorname{gap}(p)$

(LCN'05) 
We now prove the upper bound of $\operatorname{gap}(p, A S)$. Assume that $p_{0}$ is a point in the intersection of sensing void $S V_{1}$ and block $Q$ such that $\operatorname{gap}\left(p_{0}, A S\right)=\max \{\operatorname{gap}(p, A S) \mid p \in$ $\left.\cup_{1 \leq i \leq q} S V_{i}\right\}$, and $\operatorname{gap}\left(p_{0}, A S\right)=\operatorname{distance}\left(p_{0}, s_{0}\right)=r+r_{s}$, illustrated in Fig.4 (a) and (b) for $S V_{1} \cap Q=S V_{1}$ and $S V_{1} \cap$ $Q \subset S V_{1}$ respectively. The rest is to estimate the value of $r$. To do so, we draw a circle $C$ centered at $p_{0}$ with radius $r$, and we show that $C$ is entirely contained by the sensing void $S V_{1}$ by contradiction. Let us consider case (a). If $C$ is not entirely contained by $S V_{1}$ as shown in Fig.4(c), there is at least one point $p^{\prime}$ in $C$ such that $p^{\prime}$ is covered by at least one sensor $s_{0}^{\prime}$ in $A S$. Thus, distance $\left(p_{0}, s_{0}^{\prime}\right)=\overline{p_{0} p^{\prime}}+\overline{p^{\prime} s_{0}^{\prime}}<\overline{p_{0} p}+\overline{p s_{0}}=$ distance $\left(p_{0}, s_{0}\right)=\operatorname{gap}\left(p_{0}, A S\right)$, which contradicts the fact that $s_{0}$ not $s_{0}^{\prime}$ in $A S$ is the nearest active sensor to $p_{0}$. The discussion for case (b) is similar, and omitted.

We now show that $r \leq 2 d \sqrt{\frac{1-\theta}{\pi}}$. Let $p_{1}$ be a point located at a corner of $Q$ and $s$ a sensor such that the distance $\left(p_{1}, s\right)=$ $r_{1}+r_{s}$, and the area of the sector $C_{1}=\widehat{a p_{1} b}$ centered at $p_{1}$ with radius $r_{1}$ is equal to the area of $C \cap Q$. We further suppose that $p_{1}^{\prime}$ is the point of the intersection of line $\overline{p_{1} s}$ and $C_{1}$, as shown in Fig.4(d). Clearly, $r \leq r_{1}$ since $\frac{\pi r^{2}}{4} \leq \mathcal{C}(C \cap Q)=\mathcal{C}\left(C_{1}\right)=$ $\frac{\pi r_{1}^{2}}{4}$ in both cases (a) and (b). Furthermore, $r_{1} \leq 2 d \sqrt{\frac{1-\theta}{\pi}}$ since $\frac{\pi r_{1}^{2}}{4}=\mathcal{C}\left(C_{1}\right)=\mathcal{C}(C \cap Q) \leq(1-\theta) d^{2}-\sum_{i=2}^{q} \mathcal{C}\left(S V_{i} \cap\right.$ $Q)-\mathcal{C}\left(\left(S V_{1}-C\right) \cap Q\right) \leq(1-\theta) d^{2}$. Thus, the claim (1) follows. From the definition of $\operatorname{gap}_{S}$, then (2) follows.

For the connected, evenly $\theta$-coverage problem, it is often desirable to restrict the gap of each sensing void relative to $A S$ within a given threshold $\delta\left(\geq r_{s}\right)$ to guarantee the accuracy of the sensed data. With this given restriction on the gap and Theorem 1 , the block size of $R$ can be easily determined by the following corollary.

Corollary 1: For a given coverage guarantee $\theta$, a monitored region $R$ that has been partitioned into equal-size blocks with edge length $d$, and is evenly $\theta$-covered by $S$ with $A S$ of active sensors, a sensing range $r_{s}$ of each sensor, sensing voids $S V_{i}$, a gap threshold $\delta\left(\geq r_{s}\right)$, if $\operatorname{gap}_{S}\left(S V_{i}, A S\right)$ is no greater than $\delta$ for all $\mathrm{i}, 1 \leq i \leq q$, i.e. $\forall_{1 \leq i \leq q}\left(\operatorname{gap}_{S}\left(S V_{i}, A S\right) \leq \delta\right)$, then $d \leq \frac{\delta-r_{s}}{2} \sqrt{\frac{\pi}{1-\theta}}, 0<\theta<1$.

\section{Heuristic Algorithm}

In this section we propose a heuristic algorithm for the connected, evenly $\theta$-coverage problem. We start by introducing the notations that will be used in the description of the proposed algorithm.

Recall that $S$ is the set of sensors, $\theta$ is the coverage guarantee with $0<\theta<1, R$ is the monitored region that has been partitioned into equal-size blocks $Q_{1}, Q_{2}, \cdots, Q_{q}, q \geq 1, A S$ is the set of current active sensors, $r_{s}$ is the sensing range and $r_{c}$ is the transmission range of each sensor, $P_{u, v}$ is a path from $u$ to $v$. The newly covered area by a sensor $u$ relative to $A S$ is the area that is covered by $u$ but has not yet been covered by any sensor in $A S$, i.e. newly_covered_area $(u, A S)=$ $R \cap\left(f_{u}-\cup_{s \in A S} f_{s}\right)$. The newly covered area by path $P_{u, v}$ relative to $A S$ is the area that is covered by at least one sensor in $P_{u, v}$ but has not yet been covered by any sensor in $A S$, i.e. newly_covered_area $\left(P_{u, v}, A S\right)=R \cap$ $\left(\cup_{s \in P_{u, v}} f_{s}-\cup_{s \in A S} f_{s}\right)$. A path $P_{u, v}$ is called a candidate path from $u$ to $v$ relative to $A S$ if $v \in A S, u \notin A S$ and newly_covered_area $\left(P_{u, v}, A S\right)>0$.

\section{A. The gain of a candidate path}

To determine which candidate path should be included, a measurement called the gain of the path is defined, which will be determined by the following two conflicting optimization objectives. On one hand, it is desirable to add the nodes in $P_{u, v}$ into $A S$ such that newly_covered_area $\left(P_{u, v}, A S\right)$ is as large as possible and at the same time the number of nodes in $P_{u, v}$ is as small as possible, i.e. the average newly covered area per node in $P_{u, v}$ except $v$ is maximized. Formally, the nodes in $P_{u, v}$ are selected to be added into $A S$ if $\operatorname{gain}_{1}\left(P_{u, v}, A S\right)$ is maximized, where $\operatorname{gain}_{1}\left(P_{u, v}, A S\right)=$ $\frac{\mathcal{C}\left(\text { newly_covered_area }\left(P_{u, v}, A S\right)\right)}{\left|P_{u, v}\right|-1}$. On the other hand, it is expected to add the nodes in $P_{u, v}$ into $A S$ such that the number of blocks that are covered by nodes in $P_{u, v}$ but have not yet been $\theta$-covered by $A S$ is maximized, i.e. $P_{u, v}$ has the maximal $\operatorname{gain}_{2}\left(P_{u, v}, A S\right)=\mid\left\{Q_{j} \mid\left(Q_{j}\right.\right.$ is not $\theta$-covered by $\left.A S\right) \wedge$ $\exists_{w \in P_{u, v}}\left(\right.$ newly_covered_area $\left.\left.(w, A S) \cap Q_{j} \neq \emptyset\right)\right\} \mid$.

The defined gain $_{1}$ is the average gain of $P_{u, v}$ brought by the newly added sensors in $P_{u, v}$ in terms of covering more uncovered areas by these sensors, while gain $_{2}$ is the gain brought by $P_{u, v}$ in terms of covering more blocks that have not been $\theta$-covered. Therefore, a good heuristic must reflect the tradeoff between these two optimization objectives. Thus, the gain of a candidate path $P_{u, v}$ is defined as $\operatorname{gain}\left(P_{u, v}, A S\right)=$ $\sigma * \operatorname{gain}_{1}\left(P_{u, v}, A S\right)+(1-\sigma) * \operatorname{gain}_{2}\left(P_{u, v}, A S\right)$, where $\sigma$ is a constant, which is the balance factor between gain $_{1}$ and gain $_{2}, 0 \leq \sigma \leq 1$. Here, $\sigma$ plays an important role

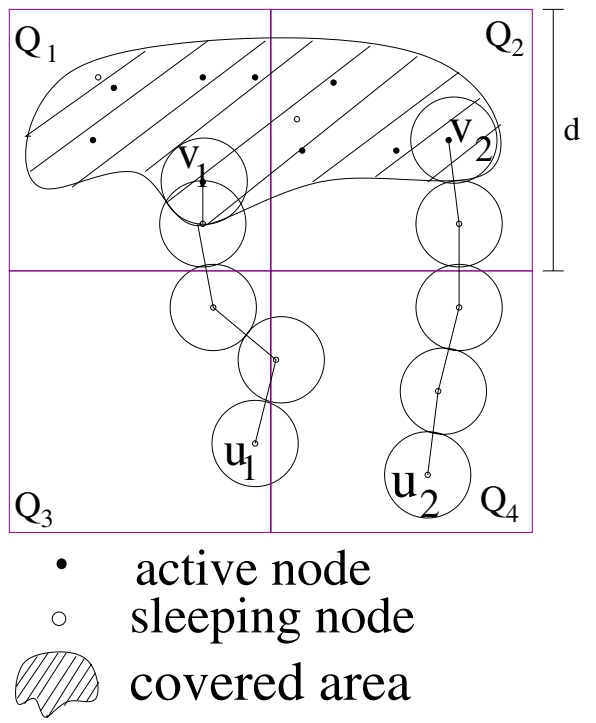

Fig. 5. The calculation of gains with $r_{s}=1$ and $r_{c}=2$.

in balancing the two optimization objectives. If $\sigma<\frac{1}{2}$, it over-emphasizes the gain $_{2}$ in terms of covering more blocks that have not been $\theta$-covered. Otherwise, gain 1 has a heavier weight. We here use an example to illustrate how to $\mathrm{c}$ (LCN'05) 
the gain of a candidate path. We assume that $r_{s}=1, r_{c}=2$, and $R$ has been partitioned into four blocks $Q_{1}, Q_{2}, Q_{3}, Q_{4}$. Assume that only $Q_{1}$ and $Q_{2}$ have already been $\theta$-covered by the current set $A S$ of active sensors as shown in Fig.5. We further assume that there are two candidate paths $P_{u_{1}, v_{1}}$ and $P_{u_{2}, v_{2}}$. Then, we have $\operatorname{gain}_{1}\left(P_{u_{1}, v_{1}}, A S\right)=2.61$ and $\operatorname{gain}_{1}\left(P_{u_{2}, v_{2}}, A S\right)=3.14$, while $\operatorname{gain}_{2}\left(P_{u_{2}, v_{2}}, A S\right)=2$ and $\operatorname{gain}_{2}\left(P_{u_{2}, v_{2}}, A S\right)=1$. If the balance factor $\sigma$ is chosen to be 0.4 , then, $\operatorname{gain}\left(P_{u_{1}, v_{1}}, A S\right)=2.06$ and $\operatorname{gain}\left(P_{u_{2}, v_{2}}, A S\right)=1.86$; otherwise, $\operatorname{gain}\left(P_{u_{1}, v_{1}}, A S\right)=2.1$ and $\operatorname{gain}\left(P_{u_{2}, v_{2}}, A S\right)=2.28$ if $\sigma$ is chosen to be 0.6 .

\section{B. Algorithm}

Due to NP-hardness of the connected, evenly $\theta$-coverage problem, the proposed algorithm will tradeoff the above two optimization objectives by using the heuristic function gain. The idea behind it is to maintain the set $A S$ of active sensors. Specifically, the algorithm proceeds in iterations. Initially, $A S$ only contains a randomly selected sensor. Within each iteration, $A S$ is updated by adding the nodes in a chosen candidate path with the maximum gain. The algorithm continues until either no nodes can be added into $A S$ or $R$ has already been connected, evenly $\theta$-covered by $A S$. The detailed algorithm follows.

Algorithm Connected_evenly_ $\theta$-Cover $\left(R, S, \theta, r_{s}, r_{c}, \sigma\right)$ Input: the monitored region $R$ that has been partitioned into blocks $Q_{1}, Q_{2}, \cdots, Q_{q}, q \geq 1$, the set $S$ of sensors, the coverage guarantee $\theta, 0<\theta \leq 1$, the sensing range $r_{s}$, the transmission range $r_{c}$, and the balance factor $\sigma$, $0 \leq \sigma \leq 1$

Output: the set $A S$ of active sensors if it exists.

begin

1. $A S=\{s\}$, where $s$ is randomly selected from $S$;

2. construct a shortest path tree $S P T$ rooted at $s$;

3. $f l a g=$ true;

4. while $($ flag $\wedge(R$ is not evenly $\theta$ - covered by $A S))$ do

5. gain $=0 ; / *$ the maximal gain $* /$

6. $\mathcal{P}=N I L ; / *$ the path with the maximal gain*/

7. for each node $u \notin A S$

8. $\quad$ compute $\operatorname{gain}\left(P_{u, v}, A S\right)$, where $v \in A S$ and $P_{u, v}$ is the path from $u$ to $v$ in $S P T$;

9. if $\left(\operatorname{gain}\left(P_{u, v}, A S\right)>\right.$ gain $)$ then

10. $\quad \operatorname{gain}=\operatorname{gain}\left(P_{u, v}, A S\right)$;

11. $\mathcal{P}=P_{u, v}$;

endif;

endfor;

12. if $($ gain $>0)$ then

13. $A S=A S \cup\{s \mid s \in \mathcal{P}\}$; else

14. flag $=$ false; /*no sensors can be added into $A S^{*} /$ endif;

endwhile;

15. if (flag) then

16. $\operatorname{return}(A S)$; endif;

end
Theorem 3: Given the coverage guarantee $\theta$ with $0<\theta<$ 1 , there is a heuristic algorithm for the connected, evenly $\theta$ coverage problem, which takes $O\left(n^{3}\right)$ time.

Proof: It can be seen that if there is a solution meeting the coverage guarantee, then, the algorithm can deliver the solution. The time complexity of the proposed algorithm is $O\left(n^{3}\right)$ due to the facts that Step 2 takes $O\left(n^{3}\right)$ time, Steps 7 and 8 take $O\left(n^{2}\right)$ time, whereas the while loop is executed at most $n$ times.

\section{Performance Evaluation}

In this section we evaluate the performance of the proposed algorithm through experimental simulations. We compare the network lifetime derived by various coverage guarantees and balance factors.

\section{A. Simulation Environment}

We assume that the monitored region $R$ is a $l \times l$ square in which the sensor nodes are deployed randomly. We further assume that $R$ is divided into $k_{1} \times k_{1}$ equal-size square blocks with the edge length of each block being $d=l / k_{1}$, where $k_{1}$ is a factor of $l$. Each block is further divided into $k_{2} \times k_{2}$ equal-size square grids with the edge length of each grid being $d^{\prime}=d / k_{2}$, where $k_{2}$ is a factor of $d$. A grid is covered by a set $S$ of sensors if the central point of the grid is contained in the sensing field $f_{s}$ of sensor $s$, where $s \in S$. A block is $\theta$-covered by a set $S$ of sensors if at least $\theta$ percentage of $k_{2} \times k_{2}$ grids of the block is covered by $S$.

The edge length of the square region $R$ is set $l=50 \mathrm{~m}$, the edge length of the block is set $d=25 \mathrm{~m}$, the edge length of the grid is set $d^{\prime}=1 \mathrm{~m}$, the number of sensors in $S$ is $1000,1500,2000,2500$ and 3000 respectively, the sensing range $r_{s}$ is 5 and the transmission range $r_{c}$ is 10, the coverage guarantee $\theta$ is set to be $0.6,0.7,0.8,0.9,0.99$ and 1.0 respectively, and the balance factor $\sigma$ is ranged from 0.4 to 0.7 with the increment of 0.1 . Each network topology with different problem size is generated using the NS-2 simulator. For each size of the network instance, the value of the network lifetime in figures is the mean of network lifetimes of 10 different network topologies.

\section{B. Coverage Guarantee Impact on Performance}

For a given network instance, the network lifetime is the number of trees, where each tree covers at least $\theta$ percentage of $R$. Each sensor node in $S$ is either "alive" or "dead". We apply the proposed algorithm to the networks in rounds. Initially, all sensor nodes in $S$ are "alive". At each round, the algorithm randomly selects a living sensor node as the root of tree $T$, and it proceeds until either the sensor nodes in $T$ can cover at least $\theta$ percentage of $R$ with minimum cardinality or no such a tree $T$ exists. After each round, if the nodes in $T$ can cover at least $\theta$ percentage of $R$, the network lifetime increases by 1 . Then, all the sensor nodes in $T$ become "dead", and $S=S-\{v \mid v \in T\}$. The algorithm terminates when all the nodes in $S$ are "dead".

We first compare the performance of the proposed algnrithm with different coverage guarantees. Fig.6 illustrated 1 
the network lifetime increases with the decline of coverage guarantee. In particular, irrespectively to the value of the balance factor $\sigma(0.4,0.5,0.6$ or 0.7$)$, the network lifetime derived by the algorithm with $\theta=0.9$ is almost twice as long as that with $\theta=1.0$, whereas the difference of their coverage accuracy is only 10 percent. This means that the network lifetime can be significantly prolonged at a relatively small expense of the accuracy. (b) The network lifetime becomes longer with the increase of the number of nodes in the monitored region.

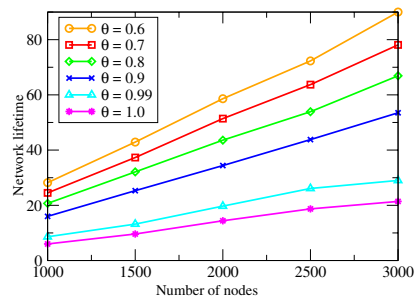

(a) $\sigma=0.4$

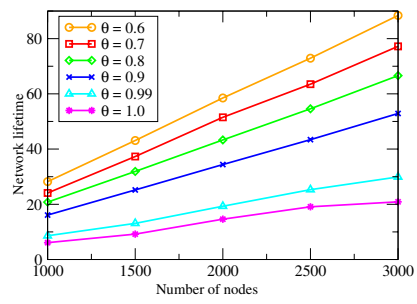

(c) $\sigma=0.6$

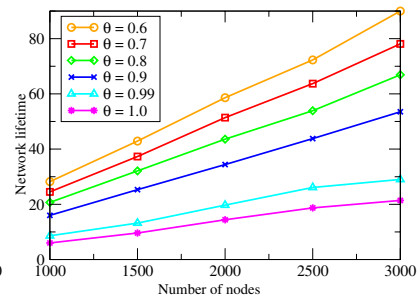

(b) $\sigma=0.5$

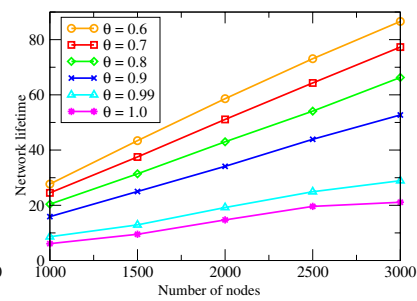

(d) $\sigma=0.7$
Fig. 6. Network Lifetime with various coverage guarantees and balance factors.

We then analyze the impact of changing rate of coverage guarantee on the performance of the proposed algorithm. Let $L(n, \theta, \sigma)$ be the network lifetime derived by the proposed algorithm when it is applied to a network instance of $n$ sensors, the coverage guarantee $\theta$, and the balance factor $\sigma$. Define $\Delta L(n, \theta, \Delta \theta, \sigma)=\frac{L(n, \theta, \sigma)-L(n, \theta+\Delta \theta, \sigma)}{\Delta \theta}$, which is the increasing rate of the network lifetime when the coverage guarantee decreases from $\theta+\Delta \theta$ to $\theta$, where $0<\theta, \theta+$ $\Delta \theta \leq 1$. Fig. 7 showed that the increasing rate is maximized when the coverage guarantee changes from 1.0 to 0.99 , where $\sigma=0.4$. That is, the maximum increment of network lifetime can be obtained when $\theta=0.99$.

\section{CONCLUSIONS}

In this paper, we first proposed the partial coverage concept in sensor networks for the first time in order to further reduce the energy consumption, thereby prolonging the network lifetime. We then analyzed the properties of partial coverage. Due to NP-hardness of the concerned problem, we instead presented a heuristic algorithm which takes into account the coverage and connectivity simultaneously. We finally con-

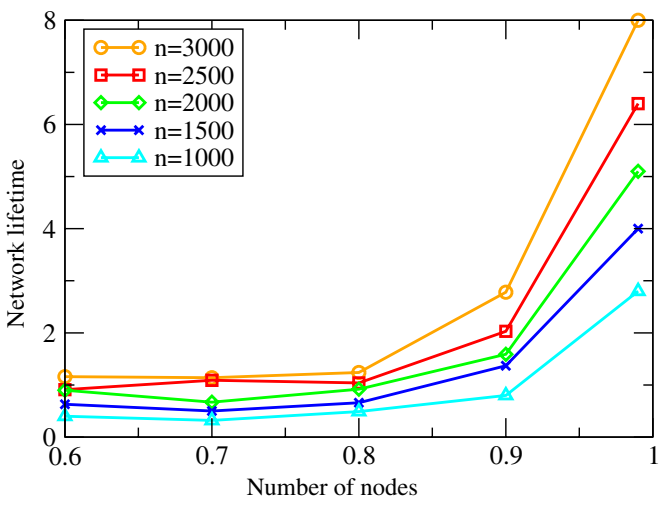

Fig. 7. The changing rate of the network lifetime.

ducted extensive experiments by simulation to evaluate the performance of proposed algorithm.

\section{REFERENCES}

[1] I. F. Akyildiz, W. Su, Y. Sankarasubramaniam and E. Cayirci, A Survey on Sensor Networks, IEEE Communications Magazine, 2002, 102-114.

[2] M. Cardei, D. MacCallum, X. Cheng, M. Min, X. Jia, D. Li, and D. -Z. Du, Wireless Sensor networks with energy effi cient organization, Journal of Interconnection Networks, Vol 3, No 3-4, 2002, 213-229.

[3] M. Cardei and D.-Z. Du, Improving Wireless Sensor Network Lifetime through Power Aware Organization, ACM Wireless Networks, Vol 11, No 3, 2005.

[4] M. Cardei and J. Wu, Energy-Effi cient Coverage Problems in Wireless Ad Hoc Sensor Networks, Computer Communications, 2005

[5] J.Carle and D. Simplot, Energy Effi cient Area Monitoring by Sensor Networks, IEEE Computer, Vol 37, No 2, 2004, 40-46.

[6] A. Cerpa and D. Estrin, Ascent: Adaptive Self-Confi guring Sensor Networks Topologies. Proc of Infocom'02, IEEE, 2002.

[7] H. Gupta, S. R. Das and Q. Gu, Connected sensor cover: Selforganization of Sensor Networks for Effi cient Query Execution. Proc. of ACM MobiHoc'03, 2003.

[8] S. Meguerdichian, F. Koushanfar, M. Potkonjak, M. Srivastava, Coverage Problems in Wireless Ad-Hoc Sensor Networks, Proc. of IEEE Infocom'01, 2001.

[9] S. Shakkottai, R. Srikant and N. Shroff, Unreliable Sensor Grids: Coverage, Connectivity and Diameter. Proc. of the 2003 IEEE Infocom'03, 2003.

[10] S. Slijepcevic and M. Potkonjak, Power Efficient Organization of Wireless Networks. Proc. of IEEE International Conference on Communications, June 2001.

[11] D. Tian and N. D. Georganas, A Coverage-Preserving Node Scheduling Scheme for Large Wireless Sensor Networks. Proc. of 1st ACM Int'l Workshop on Wireless Sensor Networks and Applications,2002.

[12] X. Wang, G. Xing, Y. Zhang, C. Lu, R. Pless, C. D. Gill, Integrated Coverage and Connectivity Confi guration in Wireless Sensor Networks. Proc. of 1st ACM Conference on Embedded Networked Sensor Systems, 2003.

[13] J. Wu and H. Li, On Calculating Connected Dominating Set for Effi cient Routing in Ad Hoc Wireless Networks. Proc.. of the 3rd International Workshop on Discrete Algorithms and Methods for Mobile /computing and Communications, 1999,7-14

[14] F. Xue and P.R. Kumar, The Number of Neighbors Needed for Connectivity of Wireless Networks. Wireless Networks, 10, 169-181, 2004.

[15] F. Ye, G. Zhong, S. Lu, and L. Zhang, Peas: A Robust Energy Conserving Protocol for Long-lived Sensor Networks. Proc. 23rd Int'l Conf. on Distributed Computing SYstems IEEE, 2003.

[16] H. Zhang and J. C. Hou, Maintaining Sensing Coverage and Connectivity in Large Sensor Networks. Proc. of the 2004 NSF International Workshop on Theoretical and Algorithmic Aspects of Sensor, Ad Hoc Wireless, and Peer-to-Peer Networks, 2004.

[17] Z. Zhou, S. Das, and H. Gupta Connected $K$-Coverage Problem in Sensor Networks, Proc. of Intl. Conf. on Computer Communications and Networks (ICCCN), 2004. 